\title{
Numerical analysis of blood flow in realistic arteries subjected to strong non-uniform magnetic fields
}

\author{
Saša Kenjereš $\check{\check{C}}^{1}$ \\ Department of Multi-Scale Physics, Faculty of Applied Sciences and J. M. Burgerscentre for \\ Fluid Dynamics, Delft University of Technology, Lorentzweg 1, 2628 CJ Delft, The Netherlands
}

\section{Abstract}

The paper reports on a comprehensive mathematical model for simulations of blood-flow under the presence of strong non-uniform magnetic fields. The model consists of a set of Navier-Stokes equations accounting for the Lorentz and magnetisation forces, and a simplified set of Maxwell's equations (Biot-Savart/Ampere's law) for treating the imposed magnetic fields. The relevant hydrodynamic and electro-magnetic properties of human blood were taken from the literature. The model is then validated for different test cases ranging from a simple cylindrical geometry to real-life right-coronary arteries in humans. The time-dependency of the wall-shear-stress for different stenosis growth rates and the effects of the imposed strong non-uniform magnetic fields on the blood flow pattern are presented and analysed. It is concluded that an imposed nonuniform magnetic field can create significant changes in the secondary flow patterns, thus making it possible to use this technique for optimisations of targeted drug delivery.

\section{Keywords}

blood flow, magnetic field, Lorentz force, magnetisation force, targeted drug delivery

\footnotetext{
${ }^{1}$ Corresponding author. Tel.:+31-15-2783649; Fax.+31-15-2782838. E-mail address: S.Kenjeres@tudelft.nl
} 


\section{Introduction}

One of the main problems of chemotherapy is often not the lack of efficient drugs, but the inability to precisely deliver and concentrate these drugs in affected areas. Failure to provide localised targeting results in an increase of toxic effects on neighbouring organs and tissues. One promising method to accomplish precise targeting is magnetic drug delivery. Here, a drug is bound to a magnetic compound injected into the blood stream. The targeted areas are subjected to an external magnetic field that is able to affect the blood stream by reducing its flow rate. In these regions the drug is slowly released from the magnetic carriers. Consequently, relatively small amounts of a drug magnetically targeted to the localised disease site can replace large amounts of the freely circulating drug. At the same time, drug concentrations at the targeted site will be significantly higher compared to the ones delivered by standard (systemic) delivery methods. Very encouraging findings have been recently reported in the clinical application of magnetic drug targeting including patients with an advanced and unsuccessfully pre-treated cancer or sarcoma, Alexiou et al. $(2000,2002,2003)$.

The most striking examples have been presented in Alexiou et al. (2005) in experiments with rabbits with artifically initiated limb tumours. It is demonstrated that treatments using only $20 \%$ of the standard drug amount with an active magnetic targeting showed full recovery of treated limbs. Drug concentration measurements in different organs 60 min after treatment, showed that treatment resulted in 26 times higher local concentrations in tumor areas, and significant reduction of drug presence in healthy organs (liver, heart, brain, kidney). In contrast to that, attempts with the standard (systemic) approach with reduction of drug dosage of $50 \%$ of normal amount were not successful.

We believe that mathematical modelling and numerical simulations can significantly contribute to further advancements of this technique. Since the key to success is associated with the possibility to deliver drugs at particular sites and with precise dosages, personalised parametric numerical studies can be performed - mimicking individual patient conditions. By specifying initial and boundary conditions in mathematical models that include the exact size and precise locations of the affected site, it will be possible to design optimised ways of drug delivery for individual patient conditions. Different sets of simulations analysing the optimal diameter of the magnetic particle carriers, can lead to optimal solutions for therapeutic drug preparations. For this purpose, a very first step is to obtain fundamental insights into underlying physics of the blood flow under 
the presence of a strong non-uniform magnetic field. This is the primary goal of the presented investigation.

Beside the magnetic drug targeting, a comprehensive mathematical model for analysis of blood flow when subjected to strong magnetic fields can be very useful in providing insights into effects of the new generation of a magnetic resonance imaging (MRI) scanners (with a magnetic field strength between 1.5 and 4 T) onto blood behaviour. Schenck et al. (1992) reported that many patients exposed to 1.5 - 4 T MRI scanning experienced sensations of nausea, vertigo, metallic taste or sleepiness during the treatments. In their in vitro experiments, Yamamoto et al. (2004) observed for the very first time that the blood viscosity (for both oxygenated and de-oxygenated blood) can be significantly increased even in a 1.5 T MRI scanner - but the underlying mechanism behind this was not fully understood.

Since we focus on numerical modelling and computer simulations of the blood flow in the presence of an external magnetic field, a short overview of numerical studies dealing with this subject is given next. Sud and Sekhon (1989) numerically studied the effects of the interactions between an imposed magnetic field and blood flow through the human arterial system. Their simplified model was based on an analytical expression for the flow rate in multi-branching arterial configuration subjected to a transversal magnetic field. Results demonstrated that the rate of blood flow through the system was reduced.

In their theoretical analysis of the interaction between magnetic field and aortic blood flow, Kinouchi et al. (1996) included the Lorentz force into the Navier-Stokes equations. A solution of of these extended Navier-Stokes equations was obtained by the finite element technique for blood flow through aortic vessels in the presence of a uniform static transversal magnetic field. Spatial distributions of the magnetically induced voltage and current densities in the aorta and surrounding tissue structure were calculated. The resulting Lorentz force led to a reduction of 5-10 \% blood volume flow at high intensities (larger of equal to $10 \mathrm{~T}$ ) of applied magnetic fields.

Bali and Awasthi (2007) analysed effects of the imposed magnetic field on the resistance to blood flow velocities in an idealised stenotic artery. They considered a steady two-dimensional axisymmetric laminar flow of a non-Newtonian fluid subjected to a transversal magnetic field. The Lorentz force was included in the momentum equations. Analytical expressions were derived for velocity and resistance and results were presented for different values of the Hartmann number $(0 \leq H a \leq 1)$. 
In analogy with the fluid dynamics of ferrofluids, Haik et al. (1999) proposed a concept for a model that describes the blood behaviour in presence of gradients of an external magnetic field. In contrast to previous studies, the Lorentz force was neglected and a magnetisation force was introduced. The same model was applied in a recent study of Khashan and Haik (2006) where numerical simulations of laminar blood flow over a two-dimensional eccentric stenotic orifice subjected to a permanent magnet were performed. The authors found that for different locations of the permanent magnet and different strengths of the imposed magnetic field, different locations of the reattachment point downstream the orifice were observed. Haik's model was applied again in Papadopoulos and Tzirtzilakis (2004) where a blood flow in a curved square duct under the influence of an applied magnetic field was studied by a finite-difference numerical method. The numerical simulations demonstrated that both axial velocity and the secondary flow at the transversal plane were significantly influenced by a strong magnetic field.

Similar results were presented in Tzirtzilakis (2005) where a blood flow in a straight rectangular duct under the influence of a uniform and a non-uniform magnetic field was numerically studied. In this model, in addition to the magnetisation force, the Lorentz force was accounted for too. It is concluded that the spatial distribution of the magnetic field plays an important role in influencing the underlying flow patterns.

In this paper we will start with an overview of the equations that constitute a comprehensive mathematical model for blood flow in the presence of strong non-uniform magnetic fields. Then these equations are discretised and solved by a finite-volume solver for general three-dimensional non-orthogonal geometries. The detailed numerical validation is started on idealised situations (horizontal cylinder), then is extended to mimic a realistic artery. For the latter purpose, a geometrical model of the human right coronary artery is developed similar to the geometrical structure (diameter, arc-length) of the artery presented in the work of Johnston et al. $(2004,2006)$. The results for a neutral (no magnetic field) situation are presented and compared to results of Johnston et al. (2004, 2006). Finally, a non-uniform magnetic field is activated and possible modulations of the local blood flow patterns will be analysed in detail. 


\section{Equations and Mathematical Model}

The equations describing a laminar incompressible flow of a Newtonian and electrically conducting bio-fluid (such as blood), subjected to external electro-magnetic fields consist of the combined set of extended Navier-Stokes and Maxwell equations:

$$
\begin{gathered}
\nabla \cdot \mathbf{V}=0, \quad \nabla \cdot \mathbf{B}=0, \quad \nabla \cdot \mathbf{J}=0 \\
\frac{\partial \mathbf{V}}{\partial t}+(\mathbf{V} \cdot \nabla) \mathbf{V}=\nu \nabla^{2} \mathbf{V}+\frac{1}{\rho}[-\nabla P+\underbrace{}_{\mathbf{F}^{\mathbf{L}} \times \underbrace{\mathbf{J}}_{\mathbf{F}^{\mathbf{M}}}+\underbrace{\mu_{0}(\mathbf{M} \cdot \nabla) \mathbf{H}}_{0}]}] \\
\nabla \times \mathbf{H}=\mathbf{J}, \quad \mathbf{J}=\sigma(\mathbf{E}+\mathbf{V} \times \mathbf{B})=\sigma(-\nabla \Phi+\mathbf{V} \times \mathbf{B}) \\
\nabla^{2} \Phi=\nabla \cdot(\mathbf{V} \times \mathbf{B})
\end{gathered}
$$

where $\rho, \sigma$ and $\nu$ are density, electric conductivity and kinematic viscosity of the working fluid. $\mathbf{H}, \mathbf{J}, \mathbf{E}, \mathbf{B}$ are the magnetic field intensity, total electric current, electric field and magnetic induction, respectively.

In the momentum equation (Eq. 2) there are two kinds of body forces caused by imposed electromagnetic fields which act on the bio-magnetic fluid (blood): the Lorentz force $\left(\mathbf{F}^{\mathbf{L}}\right.$, caused by electric conductivity of the fluid moving through an imposed magnetic field) and the magnetisation force $\left(\mathbf{F}^{\mathbf{M}}\right.$, bio-fluid magnetisation response- attraction or repulsion - due to the non-uniformity of the imposed magnetic field), Tzirtzilakis (2005). In blood vessels with diameters exceeding $10^{-4}$ $\mathrm{m}$ blood can be regarded as practically homogeneous because the scales of the microstructures (with typical diameters of $8 \times 10^{-6} \mathrm{~m}$ for red and white cells and $2-4 \times 10^{-6} \mathrm{~m}$ for platelets) are much smaller than that of flow, Pedley (1980), Ku (1997). It is generally accepted that blood behaves as a Newtonian fluid at shear rates above 100 1/s, Pedley (1980), Berger and Jou (2000).

However, in time-dependent studies mimicking an entire cardiac cycle, there are periods of time where the shear rate is below this 100 1/s limit, implying that non-Newtonian effects start to be important. In a recent study on simulations of a steady blood flow in coronary arteries, Johnston et al. (2004), have compared five different non-Newtonian blood viscosity models (Newtonian, Carreau, Walburn-Schneck, Power Law, Casson and Generalised Power Law models) and found out that the non-Newtonian effects were important only for low inlet velocities $(R e<50)$. In 
a follow-up study, addressing non-Newtonian effects in transient simulations, the same authors concluded that the non-Newtonian model is only significant for approximately $30 \%$ of the cardiac cycle, Johnston et al. (2006). These periods with non-Newtonian effects have been based on simulations with heart beat rate for a person at rest with relatively low inlet velocities - so these periods will be significantly reduced with further elevation of the heart beat rate. Based on these findings, we will use a Newtonian viscosity based blood model that represents a good first approximation for practical applications since our primary target is to focus on the magnetic field effects. Simulations using more advanced non-Newtonian models will be reported in a later publication.

Under the assumption that the magnetisation $(\mathbf{M})$ and the magnetic field $(\mathbf{H})$ are parallel, the magnetisation force can be written as $\mathbf{F}^{\mathbf{M}}=\mu_{0} M \nabla H$, where $M=|\mathbf{M}|$ and $H=|\mathbf{H}|$. The intensity of magnetisation in a bio-fluid generally depends on its temperature and density as well as on the magnetic field intensity. Accordingly, different magnetisation models are proposed in literature in order to take into account these parameters. The most elaborate mathematical model of the magnetisation is expressed by the Langevian function, $L(\xi)$ :

$$
M=n \cdot m \cdot L(\xi)=n \cdot m\left(\operatorname{coth} \xi-\frac{1}{\xi}\right), \quad \xi=\frac{\mu_{0} m H}{\kappa_{B} T}
$$

where $n, m, \kappa_{B}, T$ are the number of particles per unit volume, the magnetic moment of a particle, the Boltzmann constant and the absolute temperature, respectively, Berkovsky et al. (1993), Odenbach (2002). Here, we adopted a simplified model for the magnetisation since the considered problem does not involve any temperature changes:

$$
M=\chi H
$$

where $\chi$ is the magnetic susceptibility, Berkovsky et al. (1993), Rosensweig (1997). It is experimentally determined that in the presence of static magnetic fields the magnetic susceptibility of blood strongly depends of its local conditions, i.e. oxygenated blood behaves as a diamagnetic $\left(\chi^{\text {oxyg }}=-6.6 \times 10^{-7}\right)$ and de-oxygenated blood behaves as a paramagnetic $\left(\chi^{\text {deoxyg }}=3.5 \times 10^{-6}\right)$ material, Haik et al. (1999). The change in the magnetic susceptibility for de-oxygenated and oxygenated blood is caused by the binding of oxygen to the blood protein hemoglobin, which is responsible for transport of oxygen within a human body.

Similarly, the electrical conductivity of blood in animals and humans (in contrast to other tissues of body) has a significant value and the effects of Lorentz force caused by blood stream 
through an imposed magnetic field should be taken into account. Experiments presented in literature demonstrated that the electrical conductivity of blood depends on its velocity, i.e. the electrical conductivity of moving blood is higher than for the stationary one, Hoetink et al. (2004), Balan et al. (2004). This increase of the electrical conductivity is caused by reorientation of erythrocytes (red blood cells) influenced by the viscous forces, Fujii et al. (1999). Visser (1989) experimentally determined that compared to stationary blood, the electrical conductivity of flowing blood increased by 10, 15 and 20\% (averaged) for packed cell values (or hematrocit) of 36.4, 47.5 and $53.7 \%$, respectively. The measured electrical conductivity of flowing blood was in $0.7 \leq \sigma \leq 0.9$ $\mathrm{S} / \mathrm{m}$ range. This implies that the Lorentz force in the momentum equation should be included as demonstrated in Tzirtzilakis (2005). However, in the model of Tzirtzilakis (2005), the electric potential term was not taken into account in the expression for the total current density, i.e. the $-\nabla \Phi$ term was neglected in Eq.3. It is experimentally observed that an imposed magnetic field alternated the electrocardiogram (ECG) of the human cardiac rhythm, indicating the significance of the induced voltages, Jehenson et al. (1988), Tenforde (1992). It is also numerically demonstrated that the flow of blood in the presence of a magnetic field gives rise to electric currents in the major arteries of the central circulatory system, Kinouchi et al. (1996). In order to make the mathematical model for the blood flow behaviour in the presence of an external magnetic field as general as possible, we included both conductive and inductive currents into our model in accordance with our previous research dealing with magnetohydrodynamics (MHD) phenomena, Kenjereš and Hanjalić (2000), Hanjalić and Kenjereš (2001), Kenjereš and Hanjalić (2004), Kenjereš et al. (2004).

When a magnetic field is applied to flowing blood, additional reorientation of the erythrocytes (red blood cells, RBC) takes place. Higashi et al. (1993) found that within the magnetic field the erythrocytes are oriented with their disk plane parallel to the magnetic field direction while outside the magnetic field they show no particular orientation. As a result of this reorientation the viscosity of blood changes. Haik et al. (2001) experimentally determined that the blood flow rate decreased by $30 \%$ when subjected to a high magnetic field of $10 \mathrm{~T}$. They connected this decrease in the blood flow rate with an increase in the apparent viscosity caused by the applied magnetic field. A qualitative interpretation is provided in terms of the apparent viscosity $\left(\mu^{*}\right)$ as $\mu^{*} / \mu \propto L^{4}(\xi) / \xi^{1 / 7}$ due to the magnetic torques exerted on the erythrocytes. In the present study, we used the values of the apparent viscosity increase as proposed by Haik et al. (2001). A short summary of all blood properties is given in Table 1. 
In addition to all blood properties and the magnetisation force, the Lorentz force should be calculated too. In this work we solved a simplified set of Maxwell's equations by solving a single equation for the electric potential (induction-less assumption), originating from Ohm's law for a moving conductive medium, Eq. 4, Kenjereš and Hanjalić (2000,2004), Hanjalić and Kenjereš (2001). In order to impose magnetic field distributions, a simple procedure is used for the generated magnetic field around a series of infinitely long straight wires (Biot-Savart/Ampere's laws):

$$
B=\frac{\mu_{0} I}{2 \pi R} \rightarrow B_{x}=-\mu_{0} \sum_{i=1}^{N} \frac{I_{i}\left(y-y_{i}^{c}\right)}{\left(x-x_{i}^{c}\right)^{2}+\left(y-y_{i}^{c}\right)^{2}}, \quad B_{y}=\mu_{0} \sum_{i=1}^{N} \frac{I_{i}\left(x-x_{i}^{c}\right)}{\left(x-x_{i}^{c}\right)^{2}+\left(y-y_{i}^{c}\right)^{2}}, \quad B_{z}=0
$$

Then, the modulus of the magnetic field intensity is calculated as:

$$
B(x, y, z)=\sqrt{B_{x}^{2}+B_{y}^{2}+B_{z}^{2}}
$$

where $N$ is the number of wires, $R$ is the distance from the wire centre, $I$ is the current intensity and $\mu_{0}$ is the magnetic permeability in vacuum $\left(4 \pi \cdot 10^{-7} \mathrm{H} / \mathrm{m}\right)$. It is noted that any other magnetic field distribution (permanent magnets, superconducting magnets, realistic MRI scanner fields) can be easily incorporated in the here presented mathematical model. A complete overview of the mathematical model is given in Appendix (I).

\section{$3 \quad$ Numerical Method}

The model equations presented in the previous section are solved using an in-house developed finite volume second-order Navier-Stokes / Maxwell solver for three-dimensional flows in structured multi-block non-orthogonal geometries, Kenjereš and Hanjalić (2001,2004,2007a,2007b). The parallel execution is based on the domain-decomposition technique utilising MPI directives. Cartesian vectors and tensors components in collocated grid arrangement are applied for all variables. The second-order QUICK scheme is applied for the discretisation of the convective terms in the momentum equations. A second-order central difference scheme is applied for the diffusive terms. A fully implicit time integration, based on three-consecutive time step values, is used for the time-dependent terms. 


\section{Results and Discussion of Numerical Simulations}

\subsection{Steady blood flow through a cylinder subjected to strong non- uniform magnetic fields}

In order to validate our implementation of the extended set of Navier-Stokes equations with additional electromagnetic forcing and to demonstrate principles behind fluid flow reorganisation, a simplified flow through an infinitely long cylinder with a diameter corresponding to a typical artery size $(R e \approx 500)$ is considered first, Figs. 1,2 . The numerical mesh in the vertical plane consists of $82 \times 82 \mathrm{CVs}$ strongly clustered in the near-wall region in order to properly resolve velocity gradients. Different configurations of imposed magnetic field are analysed for both deoxygenated and oxygenated blood. The cylinder wall is treated as a non-moving electrically insulated boundary (i.e. $\partial \Phi / \partial x_{n}=0$, where $x_{n}$ is the normal to the cylinder wall).

Under the influence of a uniformly distributed (in the vertical direction) strong magnetic field $(|B|=10 \mathrm{~T})$ the flow of de-oxygenated blood is just slightly affected by the Lorentz force (in analogy with standard Hartmann flow), since the magnetisation force is equal to zero, Fig. 1-left. This is expected since the simulated working regime $(R e=525)$ is characterised by relatively small values of $H a=4$ and $N=0.03$. The absence of any secondary motion is clearly shown. This situation dramatically changes when non-uniform magnetic field distributions are imposed. A characteristic situation with a magnetic field created from a single current carrying wire located in the central vertical plane (under an angle of $0^{0}$ ) at a distance of $5 \mathrm{~mm}$ from the blood vessel wall is shown in Fig. 1-right. Now, the magnetisation force takes over the Lorentz force and strong secondary motions are generated. For this particular configuration and $R e=525$, the intensity of these secondary motions (horizontal and velocity components) is $3 \%$ of the bulk streamwise velocity. Also imprints in streamwise velocity contours are clearly visible. It is important to note that these effects are significantly smaller for oxygenated blood since the magnetic susceptibility is smaller than for de-oxygenated state (not shown here). Interesting secondary flow patterns emerge when multiple wire fields are imposed, Fig. 2. For the situation with two wires located under angles of $\pm 45^{0}$, two outwardly directed jets are created (as clearly visible from the streamtraces patterns in the middle left of Fig. 2). Along these directions, a strong deformation of the streamwise velocity contours is observed. By adding an additional third wire $\left(\right.$ at $\left.0^{0}\right)$, the number of the secondary vortices significantly increases, but their intensity decreases, Fig. 2-right. For 
the two and three wire cases, the intensity of the secondary motion reaches $6 \%$ and $3.5 \%$ of the bulk streamwise velocity. It is obvious that numerical simulations are the only way to estimate the precise details of modulation of the blood flow subjected to non-uniform magnetic fields. Simply adding more wires (more magnetic field sources) does not automatically lead to a more affected blood flow, since the magnetic field gradients are the main driving mechanism behind the magnetisation forces. It can be seen from the distributions of the magnetic flux lines (middle row in Fig. 2), that by adding the third wire, the magnetic field lines became more uniform in the central regions, resulting in reducing the intensity of the secondary motions. At the same time, the gradients in the proximity of the walls generate additional smaller scale secondary vortices, Fig. 2middle and below. A summary of all studied cases for de-oxygenated blood with focus on profiles of the streamwise velocity component along various cross-sections positions is shown in Fig. 3. The uniform magnetic field $(B \| y)$ configuration reduces the maximum velocity by $10 \%$. This flattening of the streamwise velocity profile corresponds to classical Hartmann solution. With non-uniform magnetic fields significant deviations from both the standard Poiseuille and Hartmann solutions are observed, Fig. 3. Particularly significant changes are occurring along all intersections (A-A, $\mathrm{B}-\mathrm{B}, \mathrm{C}-\mathrm{C})$ when a two wires configuration $\left( \pm 45^{0}\right)$ is applied.

In this section we clearly demonstrated that a non-uniform magnetic field can significantly affect the steady state blood flow in a long horizontal cylinder. The magnetisation force plays a major role in the dynamics of the secondary vortices, while the Lorentz force simply reduces the streamwise velocity component. Now, we move to more anatomically realistic geometries in order to see how these fluid-flow/magnetic field interactions will modulate blood flow when only parts of the blood vessels will be subjected to an external magnetic field. Before proceeding with fully coupled phenomena, we first present the pulsating blood flow in a geometry closely resembling the human right coronary artery in order to validate numerical results with available data in literature.

\subsection{Pulsating flow in arteries with different stenosis growth rates}

The numerical mesh for an anatomically realistic model of a human right-coronary artery with different stenosis growth rates is created by closely mimicking the X-ray angiograms presented in Johnston et al. (2004,2006) (similar artery diameters and arc-lengths are selected) and consists from $19^{2} \times 236 \mathrm{CVs}$, Fig.4. Different stenosis growth rates are created to occupy from 0 to 75 $\%$ of the artery cross-section. These growth rates are indicated as phase $0, \ldots$, phase 3 - where 
phase 0 corresponds to healthy and phase 3 to $75 \%$ clothed artery at stenosis location. A separate simulation is performed in order to obtain fully developed velocity profile at the inlet, starting from a uniform distribution of $0.1 \mathrm{~m} / \mathrm{s}$. Then, in order to mimic a realistic pulsating cycle, this fully developed profile is multiplied with a time varying forcing function. This function is obtained from recorded velocity signals in the right coronary artery of a normal 56 year old female and is presented in work of Matsuo et al. (1988), Fig. 5-above. It can be seen that the velocity signal exhibits cycles of strong acceleration and deceleration with a characteristic maximum of $0.2 \mathrm{~m} / \mathrm{s}$ at $0.85 \mathrm{sec}$ and an almost fully stagnant flow at $0.16 \mathrm{sec}$. Instantaneous distributions (at $1 \mathrm{sec}$ ) of the pressure and wall-shear-stress (WSS) ${ }^{2}$ along the artery walls for different stenosis growth rates are shown in Fig. 6. It is important to report that results are shown for a fully developed pulsating flow regimes, i.e. it was necessary to perform at least 5 cycles in order to get flow fields independent from the initial velocity input profile. It can be seen that the gradual pressure distribution along the artery wall for a neutral case changes into one with more pronounced pressure differences for the stenosis case, Fig. 6-right. This is due to an increase in blood flow resistance, caused by a smaller effective flow area that requires a higher pressure gradient in order to pump the same amount of blood as in the neutral case, Fig. 6-left.

It can be seen that the stenosis drastically changes both local flow patterns and pressure distributions and consequently wall-shear-stress distributions along the artery wall. Compared to the healthy artery, the maximum of the WSS at $0.85 \mathrm{sec}$ shows an increase of 5 to 40 times for $50 \%$ and $75 \%$ stenosis growth rates, respectively, Fig. 5. This illustrates the strongly increasing risks in the latter stage of the stenosis growth where a relatively small increase of stenosis area can lead to the critical rupture of the artery walls, Lasheras (2007). It is also interesting to note the strong time-dependency of the circumferentially averaged WSS along the artery wall, Fig. 5. The obtained values of WSS for the healthy artery show an excellent agreement with values presented in Johnston et al. (2004, 2006). As the majority of studies dealing with blood flow simulations, Johnston et al. $(2004,2006)$ used a commercial CFD code and only stenosis-free situations have been considered. The good agreement in WSS distributions between the data of Johnston et al. (2004, 2006) and data results obtained with our solver proved its numerical accuracy.

The velocity vectors in a plane crossing the stenosis location for maximal (0.85 sec) and minimal $(0.16 \mathrm{sec})$ peaks during the pulsating cycle for neutral case and for case with $50 \%$ stenosis growth rate are shown in Figs. 7,8. These figures nicely illustrate the richness of the flow patterns despite

\footnotetext{
${ }^{2}$ where the wall-shear stress (WSS) is calculated as $\tau_{W}=\left.\mu(\partial V / \partial y)\right|_{y=0}$
} 
the laminar flow regime. Regions with strong flow acceleration, decelerations, stagnant zones, recirculation, inner jets, strong reversals, 3D helical patterns- all simultaneously co-exist during a pulsating cycle. It can be seen that the stenosed artery shows more intensive flow velocity caused by reduction in characteristic artery diameter. This intensive jet-like flow changes recirculative pattern downstream of the stenosis location - especially during the maximum peak in pulsating cycle. It is interesting to observe that during the minimum peak instants the velocity vectors show reversal along the walls, Figs. 7,8 - below. This is caused by adverse pressure gradients followed by a strong flow deceleration.

After performing these preliminary simulations, verifying that the obtained WSS values during pulsating cycles were in good agreement with similar results presented by Johnston et al. $(2004,2006)$, new set of simulations is performed with active magnetic fields.

\subsection{Arterial blood flow subjected to the external strong non-uniform magnetic fields}

In the previous sections we have demonstrated the effects of an imposed non-uniform magnetic field on blood in a simple cylinder geometry, and simulations of pulsating blood flow in realistic arteries without external magnetic field. The question is now to what extent the influence of a magnetic field will be effective in a real artery geometry with a magnetic field imposed over a finite length. We performed a new series of simulation where both Lorentz and magnetisation force are activated. In addition, corrections of the blood apparent viscosity in magnetic fields are taken into account in accordance with correlations of Haik et al.(2001).

The spatial distributions of the magnetic field components $\left(B_{x}\right.$ and $\left.B_{z}\right)$ are shown in Fig. 9. It can be seen that the magnetic field is localised in such a way that the potential stenosed region will be influenced most effectively. The non-uniform magnetic field is generated from a single wire located parallel to the short straight segment at a distance of $2 \mathrm{~cm}$ from the artery walls. A comparison between the pressure fields without and with imposed magnetic field is shown in Fig. 10. It can be seen that significant local pressure changes are occurring at locations with a significant magnitude of the imposed magnetic field. In order to better illustrate local changes in the flow pattern caused by the imposed non-uniform magnetic field, two vertical cross-sections are selected and contours of the velocity components are plotted, Figs. 11, 12. 
At both cross-sections (located at the beginning and at the end of the relatively short straight segment of the stenotic artery) significant changes in secondary flow patterns can be observed when the magnetic field is activated (despite the relatively short effective length: the distance between these two locations is only $2-3 \mathrm{~cm}$ ). It is interesting to observe that the flow pattern is already altered at the first location and is additionally enhanced at the second location. The appearance of an additional vortical structure in the proximity of the artery wall created by the magnetisation force is clearly visible. These results confirm our previous conclusions in the idealised configurations, i.e. that an imposed non-uniform magnetic field can significantly effect and modulate blood-flow patterns under realistic conditions.

\section{Conclusions and outlook}

In this paper we presented a comprehensive mathematical model for description of blood flow in the presence of a strong non-uniform magnetic field. Both the Lorentz and magnetisation forces are taken into account in the momentum equations. A simplified set of Maxwell's equations (a combination of the Biot-Savart and Ampere's laws) is used to calculate imposed magnetic field distributions. The electric current distributions are calculated from the Ohm's law for electrically conductive moving media. Together with equations for conservation of mass and momentum, this makes a fully closed system of equations.

The results presented in this paper can be used to study new strategies for optimisation of strong non-uniform magnetic fields for localised cancer therapy. It is important to note that significantly weaker magnetic field can be used for magnetic drug targeting in practical circumstances. This is because of the fact that the magnetic particles enhance the magnetic suspectability by a few orders of magnitude, and in turn the magnetisation force effects will be stronger. In such cases, magnetic field strength between 1 and $1.5 \mathrm{~T}$ can be very effective. Novel drug delivery systems based on strong superconducting magnets are currently under development, Takeda et al. (2007), and our mathematical model can be useful for design and further optimisation of such systems.

Additional important applications can be associated with new generation of the MRI scanners that operate in strong magnetic field regimes $(1-4 \mathrm{~T})$. Similar numerical simulations can be performed by including multi-branching blood vessels and realistic magnetic field distributions 
that can be easily incorporated in our model.

Numerical simulations with the multi-branching blood vessels with flexible walls (the fluidstructure interactions), magnetic particles and non-Newtonian viscosity behaviour will be subject of our future investigations.

\section{Acknowledgement}

This research has been made possible by a fellowship of the Royal Netherlands Academy of Arts and Sciences (KNAW) (2005/2006), a Burgers Visiting Professorship at the Institute of Physical Science and Technology, University of Maryland, College Park, U.S.A. (2005/2006) and a Guest Professorship at the AGH University of Science and Technology, Krakow, Poland (2007).

\section{Appendix I}

The final version of the model used in this study (in index notation) is given next. The following assumptions are used: the flow is laminar, incompressible and isothermal. The working fluid is homogeneous and Newtonian. The molecular viscosity of blood in the presence of a magnetic field is calculated in accordance with Haik et al. (2001). The divergency-free condition is imposed for velocity, magnetic fields and electric current density:

$$
\frac{\partial V_{i}}{\partial x_{i}}=0, \quad \frac{\partial B_{i}}{\partial x_{i}}=0, \quad \frac{\partial J_{i}}{\partial x_{i}}=0
$$

The momentum equations extended by the Lorentz $\left(F_{i}^{L}\right)$ and the magnetisation $\left(F_{i}^{M}\right)$ forces.

$$
\frac{\partial V_{i}}{\partial t}+V_{j} \frac{\partial V_{i}}{\partial x_{j}}=\frac{\partial}{\partial x_{j}}\left(\nu \frac{\partial V_{i}}{\partial x_{j}}\right)+\frac{1}{\rho}[\frac{-\partial P}{\partial x_{i}}+\underbrace{\varepsilon_{i j k} J_{j} B_{k}}_{F_{i}^{L}}+\underbrace{\mu_{0} \chi\left|H_{i}\right| \frac{\partial\left|H_{i}\right|}{\partial x_{i}}}_{F_{i}^{M}}]
$$

The full set of the Maxwell's equations for the one-way coupled fluid flow and electromagnetic fields interactions reduces to a simple (scalar) electric potential equation:

$$
\frac{\partial^{2} \Phi}{\partial x_{i}^{2}}=\frac{\partial}{\partial x_{i}}\left(\varepsilon_{i j k} V_{j} B_{k}\right)
$$

The electric current density and magnetic field intensity are calculated from following expressions:

$$
J_{i}=\sigma\left(-\frac{\partial \Phi}{\partial x_{i}}+\varepsilon_{i j k} V_{j} B_{k}\right), \quad\left|H_{i}\right|=\frac{1}{\mu_{0}}\left|B_{i}\right|
$$




\section{References}

Alexiou, C., Arnold, W., Klein, R. J., Parak, F. G., Hulin, P., Bergemann, C., Erhardt, W., Wagenpfeil, S. and Lubbe, A. S., 2000. Loco-regional Cancer Treatment with Magnetic Drug Targeting. Cancer Research 60 (23), pp.6641-6648.

Alexiou, C., Schmidt, A., Klein, R., Hulin, P., Bergemann, C. and Arnold, W., 2002. Magnetic drug targeting: biodistribution and dependency on magnetic field strength. Journal of Magnetism and Magnetic Materials 252 (1-3), pp.363-366.

Alexiou, C., Jurgons R., Schmid R.J., Bergemann, C., Henke, J., Erhardt, W., Huenges, E. and Parak, F., 2003. Magnetic drug targeting-biodistribution of the magnetic carrier and the chemotherapeutic agent mitoxantrone after locoregional cancer treatment. J. Drug Target. 11 (3), pp.139-149.

Alexiou, C., Jurgons R., Schmid R., Erhard, W., Parak, F., Bergemann, C. and Iro. H., 2005. Magnetisches Drug Targeting - ein neuer Ansatz in der lokoregionären Tumortherapie mit Chemotherapeutika. HNO (Hals-, Nasen-, Ohren-Heilkunde) Journal 53 (7), (in German), pp. 618-622.

Balan, C., Balut, C., Gheorghe, L., Gheorghe, C., Gheorghiu, E. and Ursu, G., 2004. Experimental determination of blood permittivity and conductivity in simple shear flow. Clinical Hemorheology and Microcirculation 30, pp.359-364

Berger, S. A. and Jou, L. D., 2000. Flows in Stenotic Vessels. Annual Review of Fluid Mechanics 32, pp.347-382

Berkovsky, B.M., Medvedev, V. F. and Krakov, M. S., 1993. Magnetic Fluids: Engineering Applications, Oxford University Press

Fujii, M., Nakajima K., Sakamoto, K. and Kanai, H., 1999. Orientation and deformation of erythrocytes in flowing blood. Annals of N.Y. Acad. Sci. 873, pp.245-261

Haik, Y., Pai, V. and Chen, C.-J., 1999. Biomagnetic fluid dynamics, in the Proceedings of Fluid Dynamics at Interfaces, edited by W. Shyy and R. Narayanam, Cambridge University Press, Cambridge, pp. 439-452.

Haik, Y., Pai, V. and Chen C.-J., 2001. Apparent viscosity of human blood in a high static 
magnetic field. Journal of Magnetism and Magnetic Materials 225, pp. 180-186

Hanjalić, K. and Kenjereš, S., 2001. T-RANS simulation of deterministic eddy structure in flows driven by thermal buoyancy and Lorentz force. Flow, Turbulence and Combustion 66 (4), pp. $427-451$.

Higashi, T., Yamagishi, A., Takeuchi, T., Kawaguchi, N., Sagawa, S., Onishi, S. and Date, M., 1993. Orientation of Erythrocytes in a Strong Static Magnetic Field. Blood 82 (4), pp.1328-1334

Hoetink, A. E., Faes, T. J. C., Visser, K. R. and Heethaar, R. M., 2004. On the Flow Dependency of the Electrical Conductivity of Blood. IEEE Transactions on Biomedical Engineering $\mathbf{5 1}$ (7), pp.1251-1261

Jehenson, P., Duboc, D., Lavergne, T., Guize, L., Guerin, F., Degeorges, M. and Syrota, A., 1988. Change in human cardiac rhythm induced by a 2T static magnetic field. Radiology 166, pp.227-230.

Johnston, B.M., Johnston, P. R., Corney, S., Kilpatrick, D., 2004. Non-Newtonian blood flow in human right coronary arteries: steady state simulations. Journal of Biomechanics 37, pp.709-720

Johnston, B.M., Johnston, P.R., Corney, S., Kilpatrick, D., 2006. Non-Newtonian blood flow in human right coronary arteries: transient simulations. Journal of Biomechanics 39, pp.1116-1128

Kenjereš, S. and Hanjalić, K., 2000. On the implementation of effects of Lorentz force in turbulence closure models. Int. J. Heat and Fluid Flow 21 (3), pp. 329-337.

Kenjereš, S. and Hanjalić, K., 2004. Numerical simulation of magnetic control of heat transfer in thermal convection. Int. J. Heat and Fluid Flow 25 (3), pp. 559-568.

Kenjereš, S., Hanjalić, K. and Bal, D., 2004. A direct-numerical-simulation-based secondmoment closure for turbulent magnetohydrodynamic flows. Physics of Fluids 16 (5), pp.12291241.

Kenjereš, S. and Hanjalić, K., 2007a. Numerical simulation of a turbulent magnetic dynamo. Physical Review Letters 98 (10), Art. No. 104501, pp.1-4.

Kenjereš, S. and Hanjalić, K., 2007b. Numerical insights into magnetic dynamo action in a turbulent regime. New Journal of Physics 9, Art. No. 306, pp. 1-29. 
Khashan, S.A. and Haik, Y., 2006. Numerical simulation of biomagnetic fluid downstream an eccentric stenotic orifice. Physics of Fluids 18 (11), 113601.

Kinouchi, Y., Yamaguchi, H. and Tenforde, T. S., 1996. Theoretical analysis of magnetic field interactions with aortic blood flow. Bioelectromagnetics 17, pp.21-32.

Ku, D. N., 1997. Blood Flow in Arteries, Annu. Rev. Fluid Mech. 29, pp. 399-434.

Lasheras, J. C., 2007. The Biomechanics of Arterial Aneurysms. Annu. Rev. Fluid Mech. 39, pp.293-319.

Matsuo, S., Tsuruta, M., Hayano, M., Imamura, Y., Eguchi, Y., Tokoshima, T. and Tsuji, S., 1988. Phasic Coronary Artery Flow Velocity Determined by Doppler Flowmeter Catheter in Aortic Stenosis and Aortic Regurgitation, The American Journal of Cardiology, Vol.62, pp.917-922

Odenbach, S. (Ed.), 2002. Ferrofluids: Magnetically Controllable Fluids and Their Applications. Lecture Notes in Physics, Springer-Verlag, Berlin-Heidelberg

Pedley, T. J., 1980. The Fluid Mechanics of Large Blood Vessels, Cambridge University Press, Cambridge.

Papadopoulos, P.K. and Tzirtzilakis, E. E., 2004. Biomagnetic flow in a curved square duct under influence of an applied magnetic field. Physics of Fluids 16 (8), pp.2952-2962.

Rekha, B. and U. Awasthi, 2007. Effect of a magnetic field on the resistance to blood flow through stenotic artery. Applied Mathematics and Computations 188, pp.1635-1641.

Rosensweig, R.E., 1997. Ferrohydrodynamics, Dover, Mineola, New York

Schenck, J. F., Dumoulin, C. L., Redington, R. W., Kressel, H. Y., Elliot, R. T. and McDougall, I. L., 1992. Human exposure to 4.0-Tesla magnetic fields in a whole-body scanner. Med. Phys. 19, pp.1089-1098.

Sud, V. K. and Sekhon, G. S., 1989. Blood flow through human arterial system in the presence of a steady magnetic field. Phys. Med. Biol. 34 (7), pp.795-805.

Takeda, S., Mishima, F., Fujimoto, S., Izumi, Y. and Nishijima, S., 2007. Development of magnetically targeted drug delivery system using superconducting magnet. Journal of Magnetism and Magnetic Materials 311, pp.367-371. 
Tenforde T. S., 1992. Interaction mechanisms and biological effects of static magnetic fields. Automedica 14, pp.271-293.

Tzirtzilakis, E.E., 2005. A mathematical model for blood flow in magnetic field. Physics of Fluids 17, Art. No. 077103, pp. 1-15

Visser, K. R., 1989. Electric conductivity of stationary and flowing human blood at low frequencies. 11th Annual International Conference on the IEEE Engineering in Medicine and Biology Society, pp.1540-1542

Yamamoto, T., Nagayama, Y. and Tamura, M., 2004. A blood-oxygenation-dependent increase in blood viscosity due to a static magnetic field. Phys. Med. Biol. 49, pp. 3267-3277. 


\section{Nomenclature}

\begin{tabular}{ll}
$R e=\frac{U D}{\nu}$ & Reynolds number $(-)$ \\
$H a=B_{0} D \sqrt{\frac{\sigma}{\rho \nu}}$ & Hartmann number (-) \\
$N=\frac{\sigma B_{0}^{2} D}{\rho U}$ & Stuart (interactive) number $(-)$ \\
$D$ & characteristic diameter $(\mathrm{m})$ \\
$I$ & electric current intensity $(\mathrm{A})$ \\
$N$ & number of wires $(-)$ \\
$P$ & Pressure (Pa) \\
$R$ & radial distance from a wire with current $(\mathrm{m})$ \\
$T$ & temperature $(\mathrm{K})$ \\
$W S S$ & wall-shear-stress $(\mathrm{Pa})$ \\
$\mathbf{B}$ & magnetic flux density $(\mathrm{magnetic}$ induction $)(\mathrm{T})$ \\
$\mathbf{E}$ & electric field intensity $(\mathrm{V} / \mathrm{m})$ \\
$\mathbf{F}^{\mathbf{L}}$ & Lorentz force $\left(\mathrm{N} / \mathrm{m}^{3}\right)$ \\
$\mathbf{F}^{\mathbf{M}}$ & magnetisation force $\left(\mathrm{N} / \mathrm{m}^{3}\right)$ \\
$\mathbf{H}$ & magnetic field intensity $(\mathrm{A} / \mathrm{m})$ \\
$\mathbf{J}$ & total current density $\left(\mathrm{A} / \mathrm{m}^{2}\right)$ \\
$\mathbf{M}$ & electric potential $(\mathrm{V})$ \\
\hline &
\end{tabular}

\section{Greek symbols}

$\rho \quad$ fluid density $\left(\mathrm{kg} / \mathrm{m}^{3}\right)$

$\nu \quad$ kinematic viscosity $\left(\mathrm{m}^{2} / \mathrm{s}\right)$

$\sigma \quad$ electric conductivity $(\mathrm{S} / \mathrm{m})$

$\mu_{0} \quad$ magnetic permeability in vacuum $(\mathrm{H} / \mathrm{m})$

$\kappa_{B}$ Boltzmann constant $(\mathrm{J} / \mathrm{K})$

$\chi \quad$ magnetic susceptability (-) 


\section{Table Captions}

Tab.1 Properties of the bio-magnetic fluid (blood) used for numerical simulations. 


\section{Figure Captions}

Fig. 1. Blood flow in a long horizontal cylinder under the presence of a non-uniform magnetic field, $R e=525, H a=4, N=0.03$ : First row- contours of $W / W_{m}$ and streamlines; Second rowcontours of $V / W_{m}$ and magnetic flux lines; Third row- contours of $U / W_{m}$ and $\left.\left|B_{0}\right|\right)$. Left- neutral case (no-magnetic field); Right- a magnetic field $\left(\left|B_{0}\right|_{\max }=10 \mathrm{~T}\right)$ created by a single wire located $5 \mathrm{~mm}$ from the cylinder wall at an angle of $0^{0}$.

Fig. 2. See caption in previous figure: A magnetic field $\left(\left|B_{0}\right|_{\max }=10 \mathrm{~T}\right)$ created by: left- two wires located $5 \mathrm{~mm}$ from the cylinder wall under angles of $\pm 45^{0}$, right- three wires located $5 \mathrm{~mm}$ from the cylinder wall under angles of $+45^{0}, 0^{0},-45^{0}$.

Fig. 3. Profiles of the non-dimensional streamwise velocity $\left(W / W_{m}\right)$ in a vertical plane along the different angles $0^{0}, 90^{\circ},-45^{0}$, respectively, $R e=525$. The $\alpha$ angle indicates location of the magnetic sources (wires) in the vertical plane.

Fig. 4. The numerical mesh $(19 \times 19 \times 236 \mathrm{CVs})$ used for simulations of blood flow through a model of a human right coronary artery with different stages of the stenosis growth: left- healthy artery, right- stenotic arthery (with $50 \%$ artery diameter reduction).

Fig. 5. Above- the time-dependency of the inlet velocity closely mimicking recorded velocity signal in the right-coronary artery of a real patient (56 years old healthy female), Matsuo et al. (1988). Below- the time-dependency of the circumferentialy averaged wall-shear-stress (WSS) distributions along the artery walls (the axial coordinate cooresponds to the non-dimensional artery arc-length value defined as $\left.L_{\text {arc }} / L_{\text {tot }}\right)$ for different stages of the stenosis growth $(0 \%, 50 \%$ and $75 \%$ ) respectively.

Fig. 6. Distributions of the pressure (-left) and the wall-shear-stress (WSS) (-right) along the artery walls for different stages of stenosis growth: a healthy artery (-above) and $50 \%$ stenosis (-below). Steady solutions obtained with an uniform inlet velocity of $U_{\text {in }}=0.1 \mathrm{~m} / \mathrm{s}, R e=500$.

Fig. 7. Velocity vectors in a plane crossing the potential stenosis location for maximal $(t=0.85$ sec, -above) and minimal ( $t=0.16 \mathrm{sec}$, -below) peaks during a pylsating cycle shown in previous Fig. - the healthy artery case. 
Fig. 8. Velocity vectors in a plane crossing the stenosis location (a case with $50 \%$ stenosis growth) for maximal ( $t=0.85 \mathrm{sec}$, -above) and minimal ( $t=0.16 \mathrm{sec}$, -below) peaks during a pulsating cycle.

Fig. 9. Contours of the magnetic field components originating from a line wire source located at $2 \mathrm{~cm}$ distance from the right coronary artery, $\left(B_{x}\right.$ - above, $B_{z}$ - below, $\left.\left|B_{0}\right|=10 \mathrm{~T}\right)$ along the artery walls.

Fig. 10. Contours of the local pressure distribution along the artery walls without (-above) and and with a magnetic field originating from a line wire source located at $2 \mathrm{~cm}$ distance from the right coronary artery, $\left(\left|B_{0}\right|=10 \mathrm{~T}\right.$, -below).

Fig. 11. Contours of the local pressure along the artery wall (-above) and contours of the W velocity indicating changes in the secondary flow patterns due to presence of an external magnetic field (with identical specification as in previous figure).

Fig. 12. Similar as in the previous figure, only now at different location $(2 \mathrm{~cm}$ upstream from the previous location) and U velocity components are shown. 
Table 1:

\begin{tabular}{ccccc}
\hline \hline$\rho$ & $\nu$ & $\sigma$ & $\chi^{\text {oxyg }}$ & $\chi^{\text {deoxyg }}$ \\
\hline $1050 \mathrm{~kg} / \mathrm{m}^{3}$ & $4 \times 10^{-6} \mathrm{~m}^{2} / \mathrm{s}$ & $0.7-0.9 \mathrm{~S} / \mathrm{m}$ & $-6.6 \times 10^{-7}$ & $3.5 \times 10^{-6}$ \\
\hline \hline
\end{tabular}



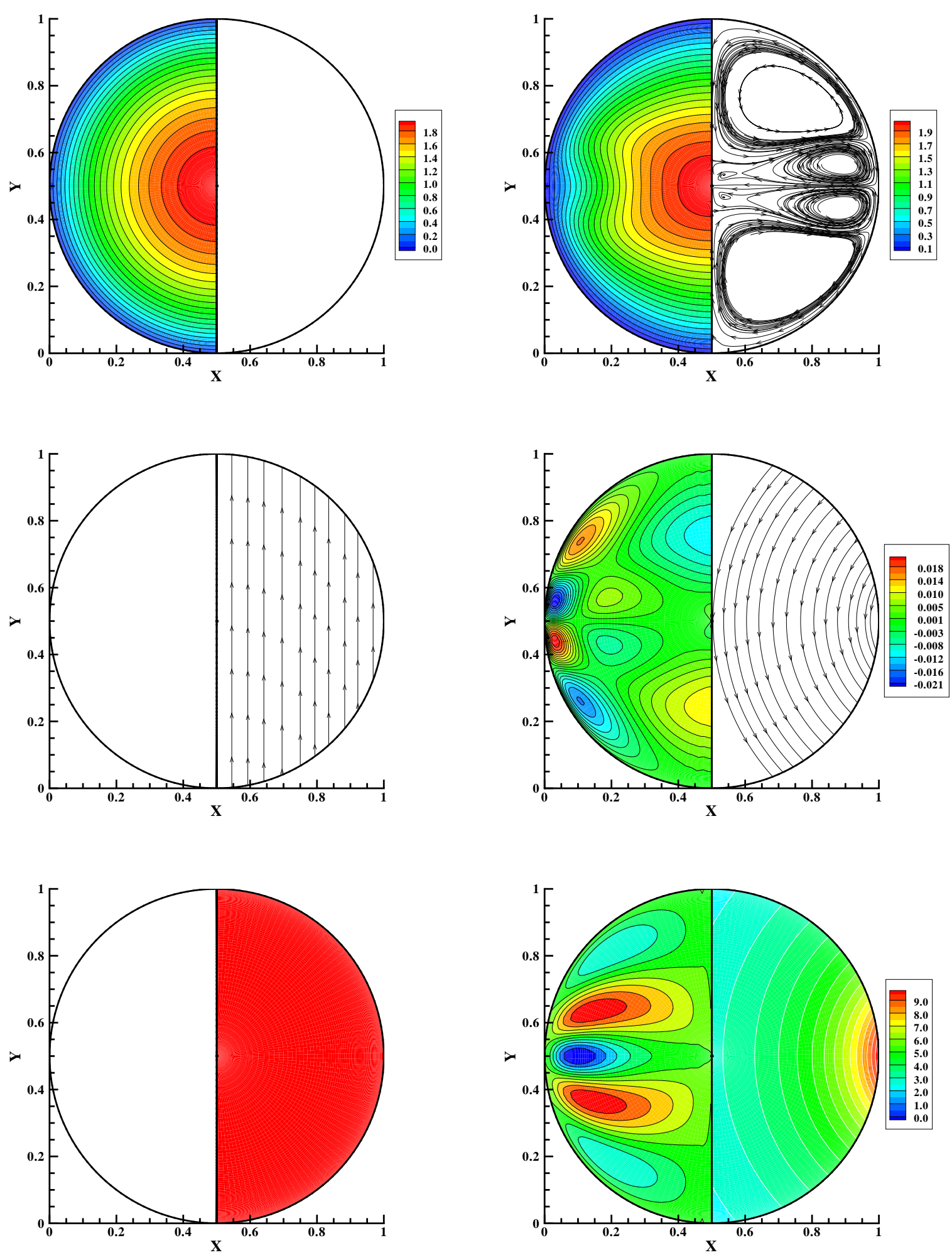

Figure 1: 

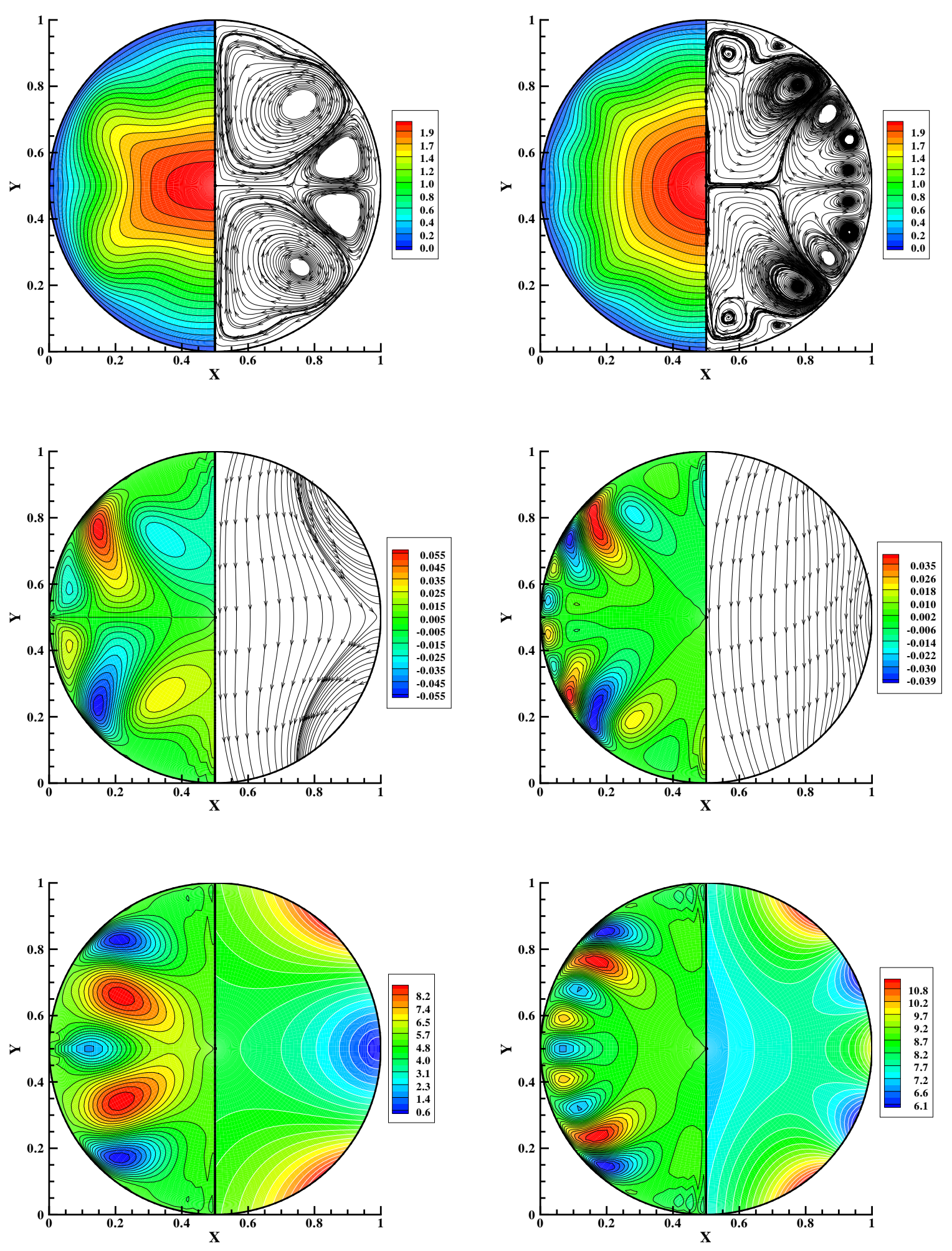

Figure 2: 

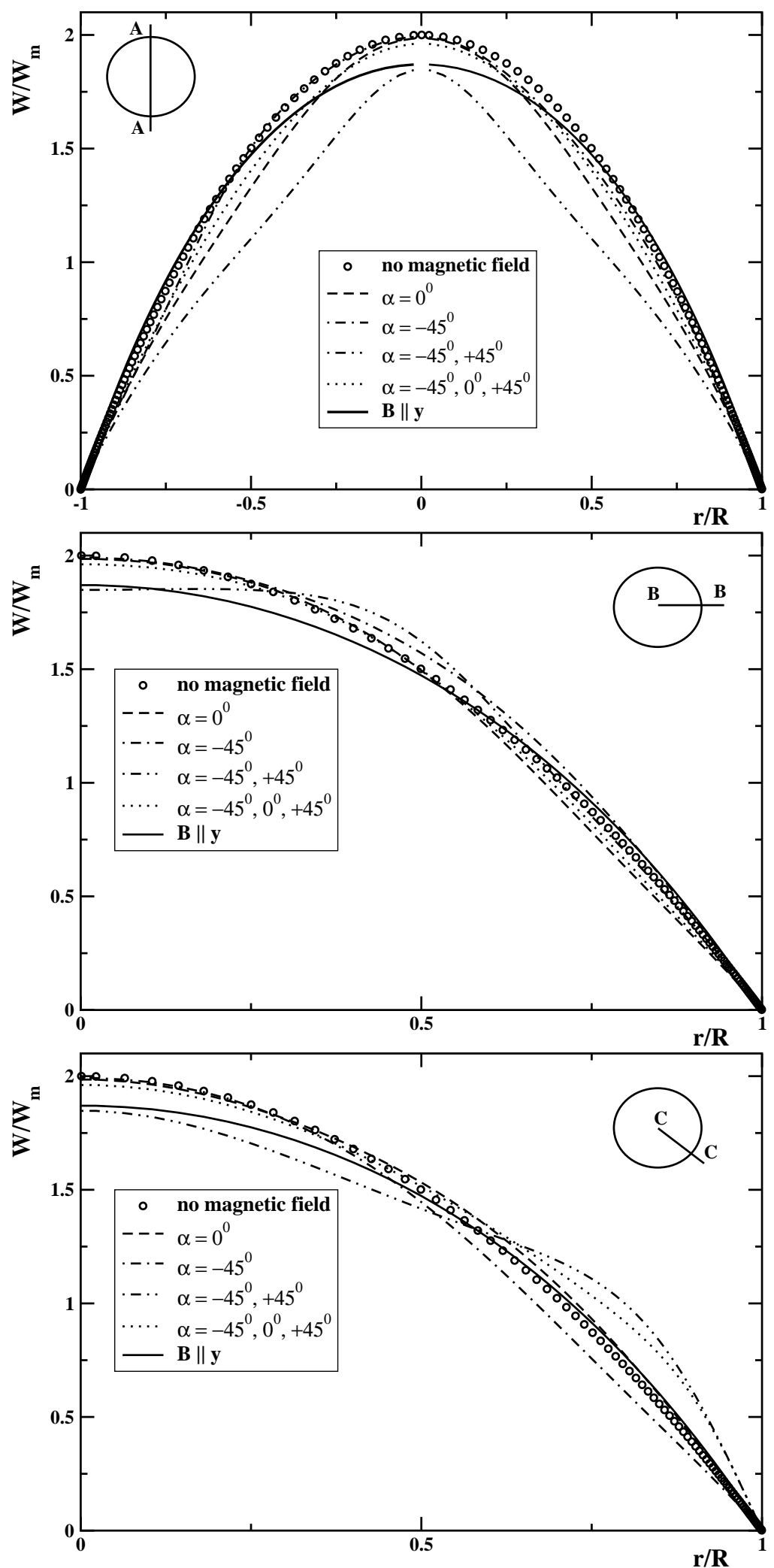

Figure 3: 

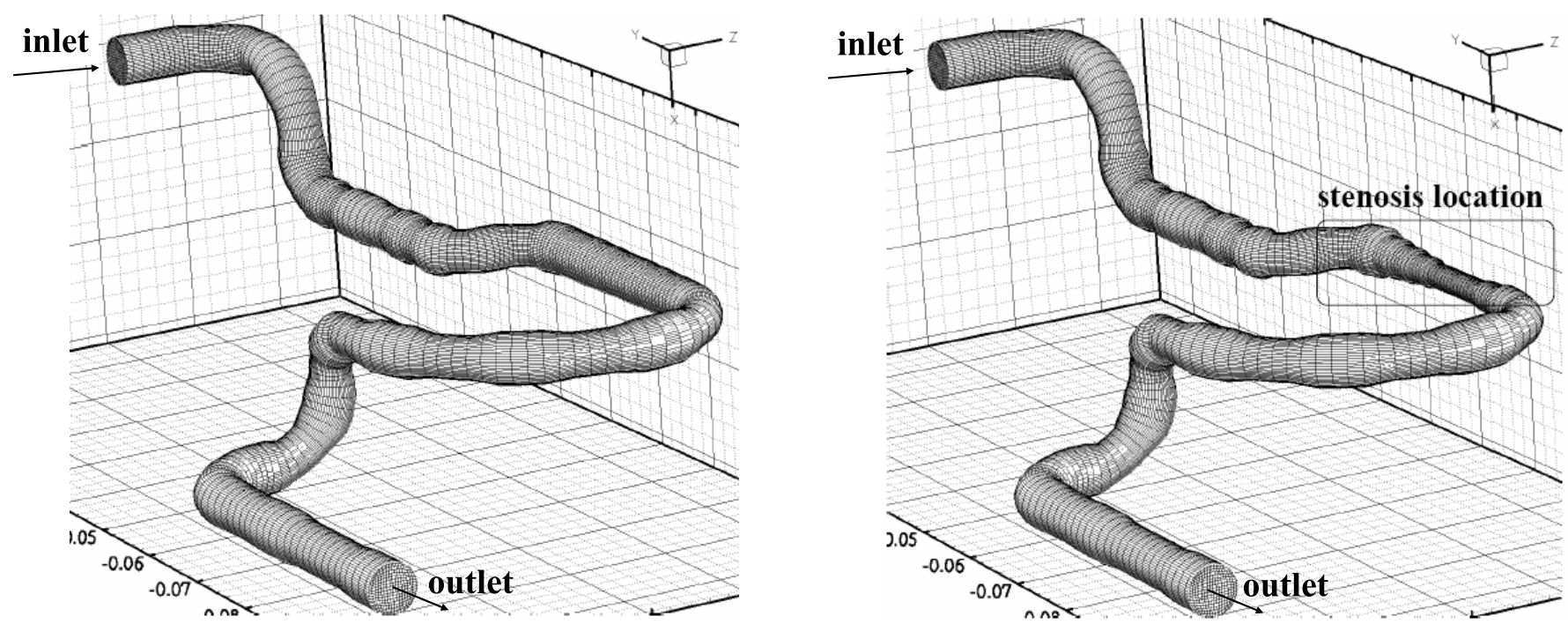

Figure 4: 

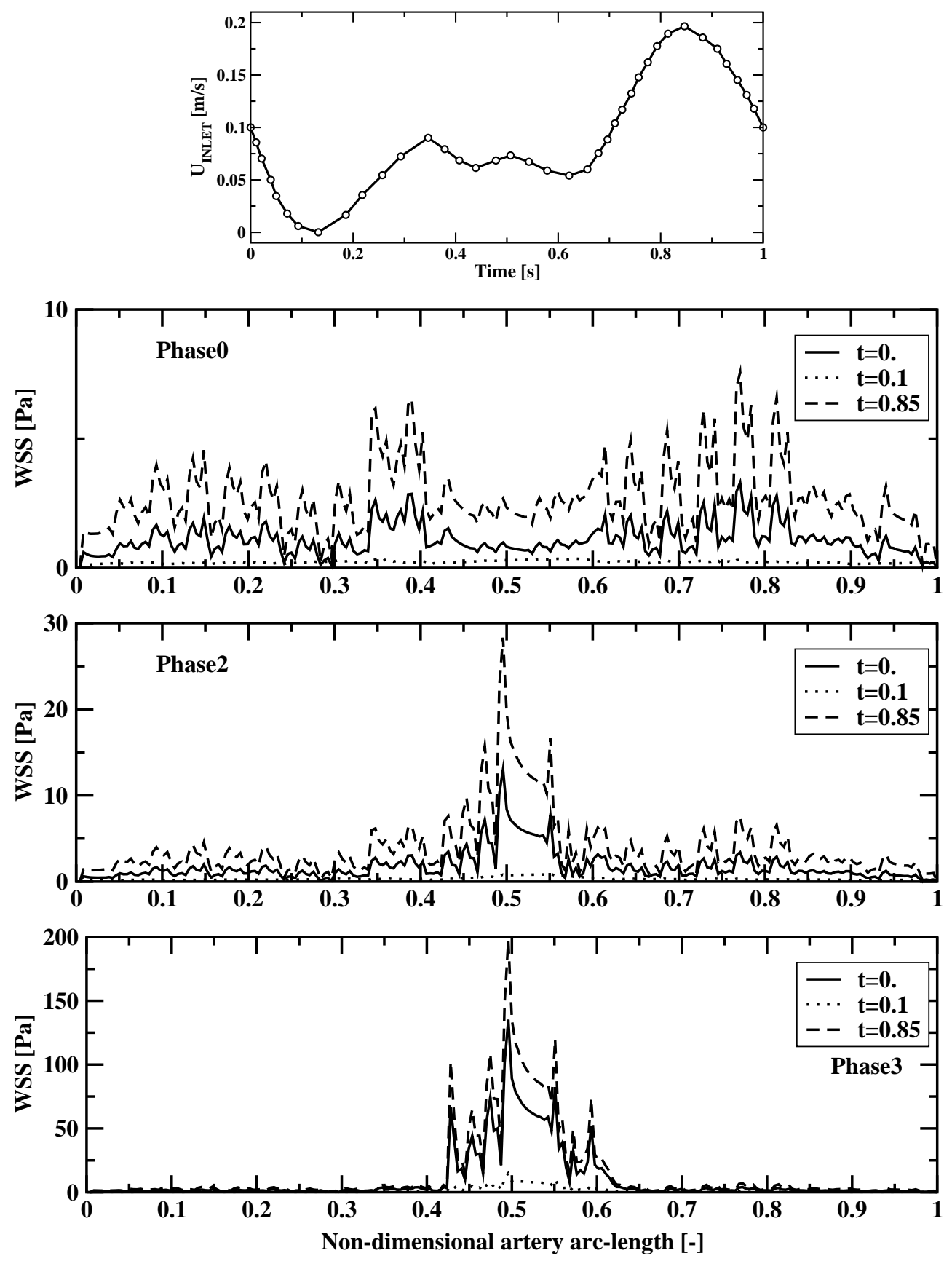

Figure 5: 

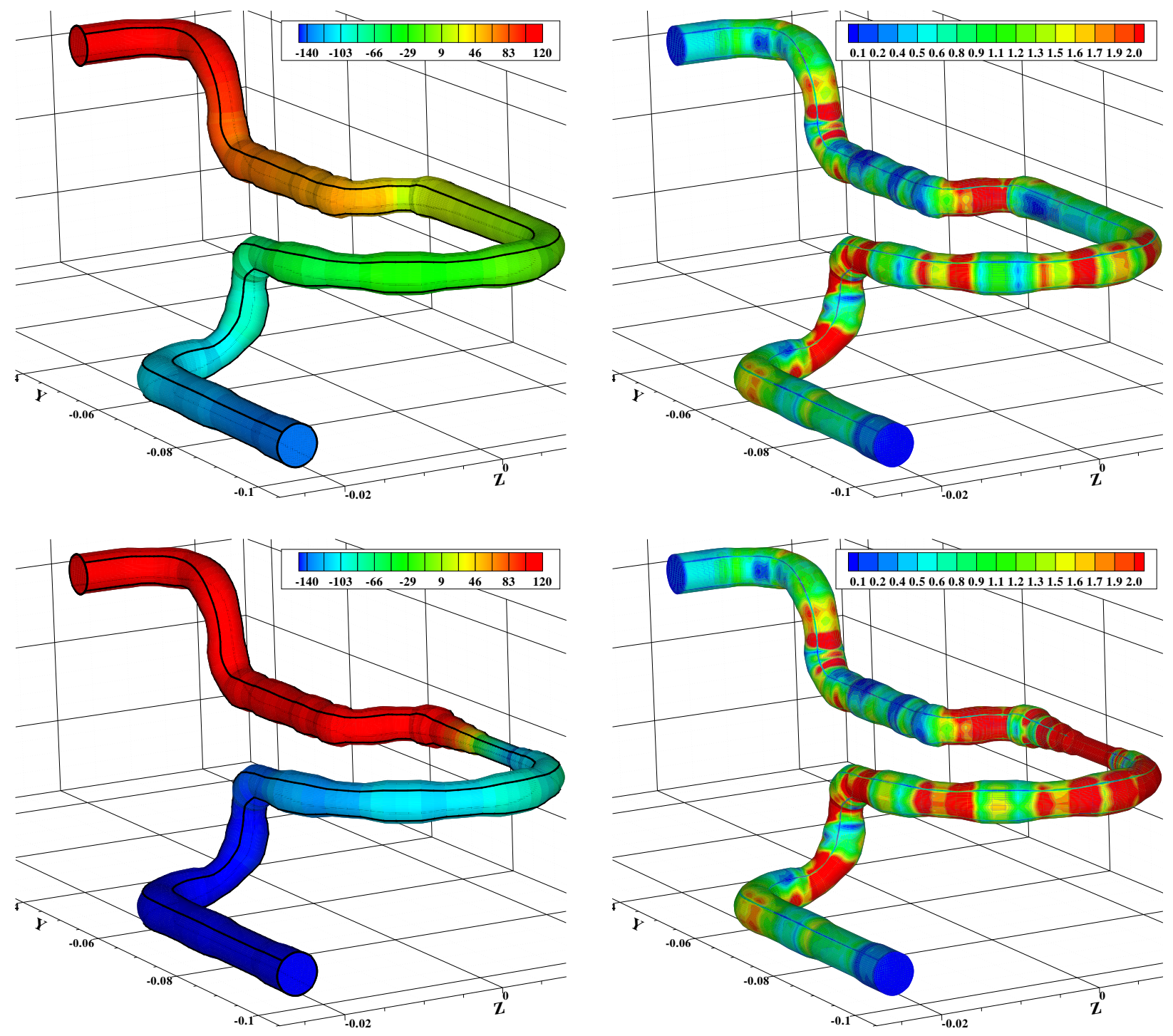

Figure 6: 

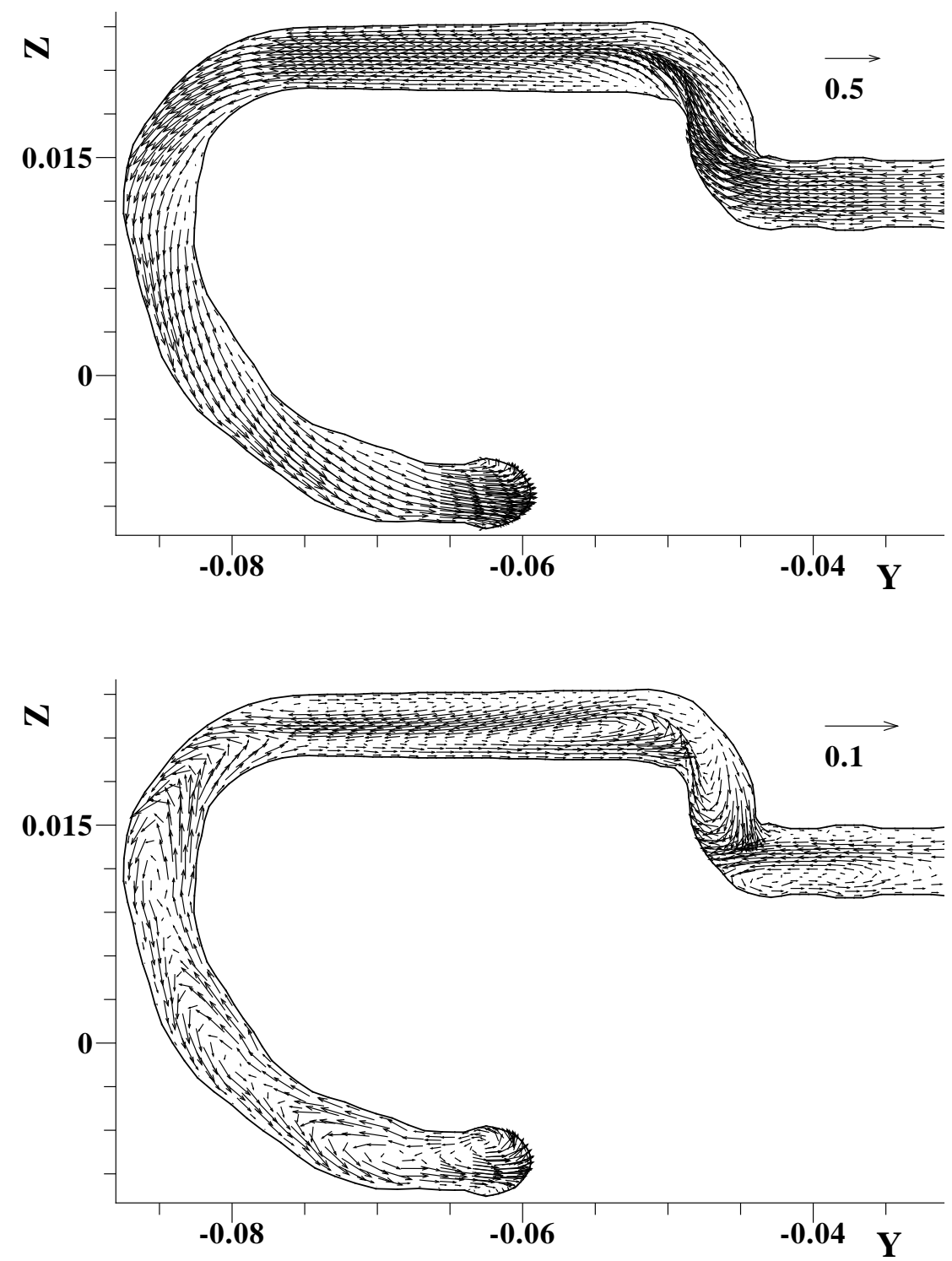

Figure 7: 

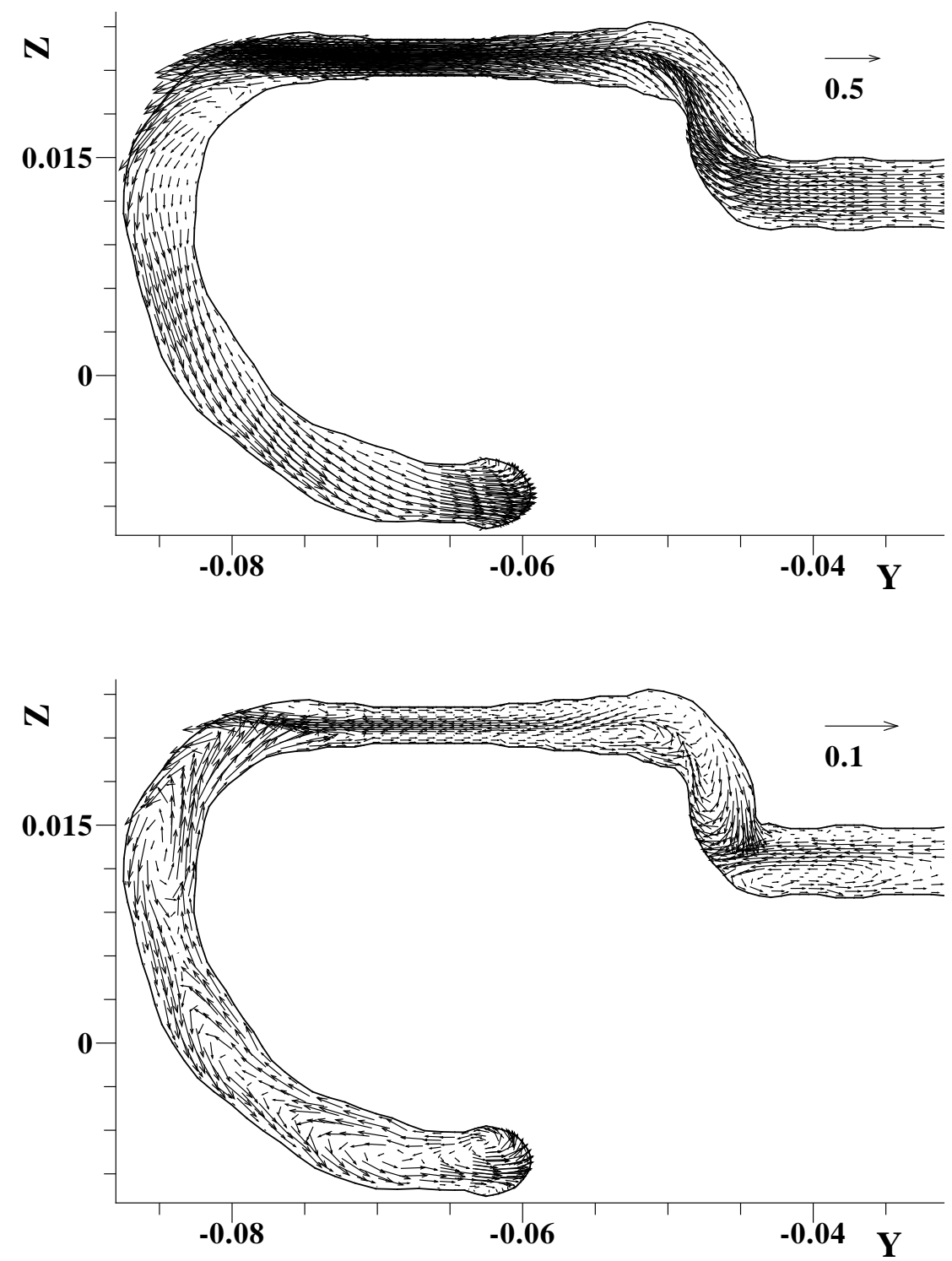

Figure 8: 

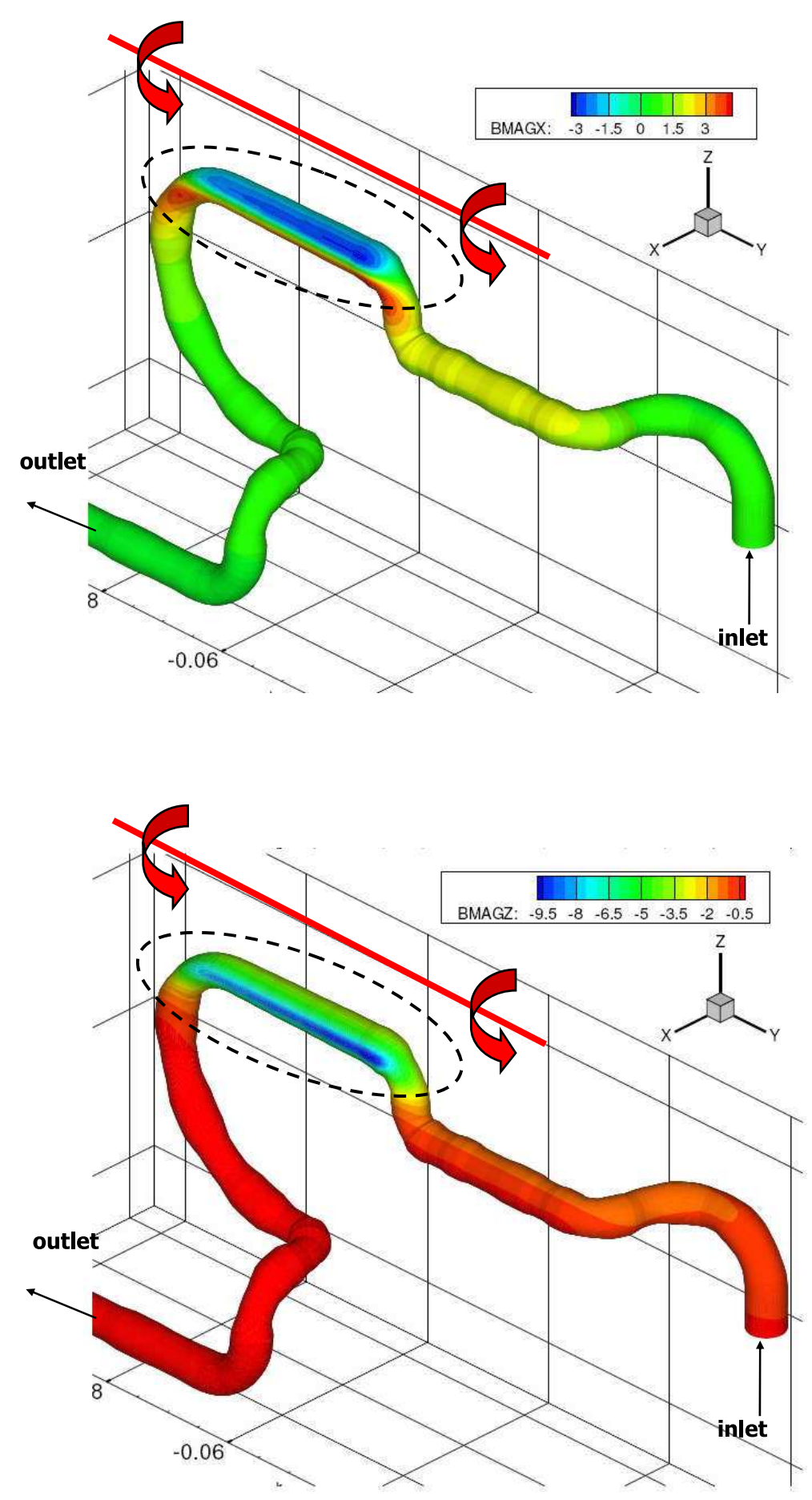

Figure 9: 

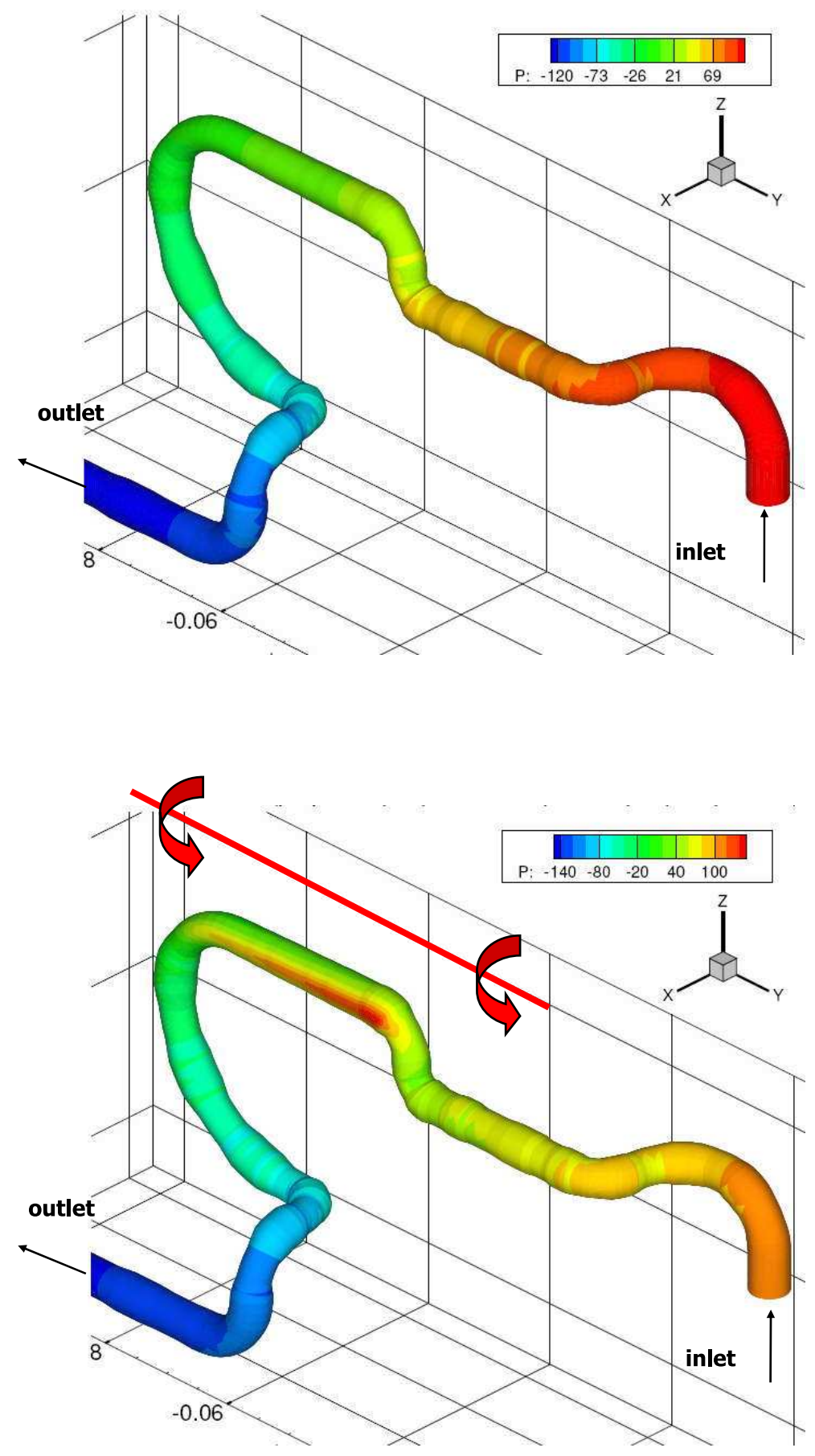

Figure 10: 


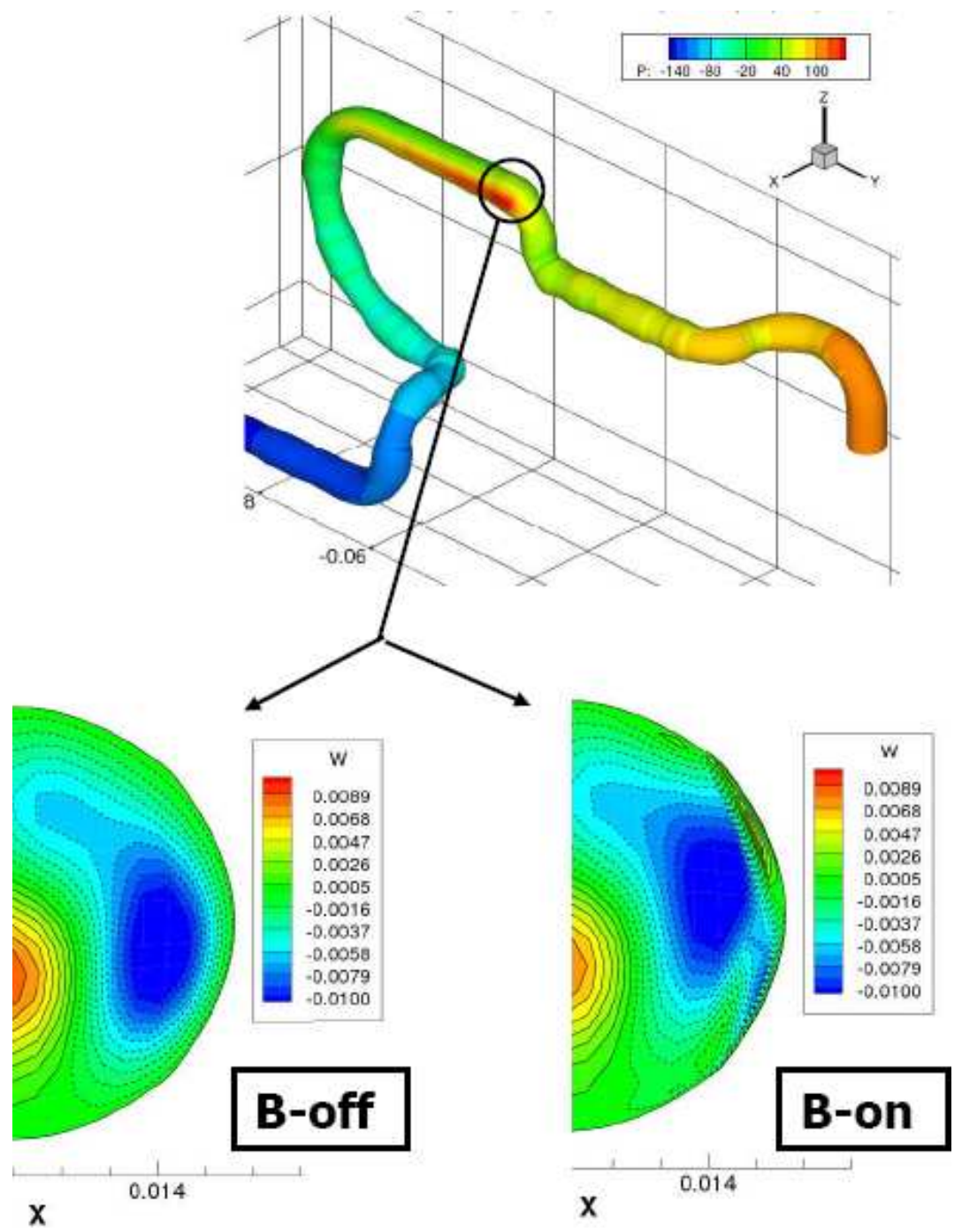

Figure 11: 


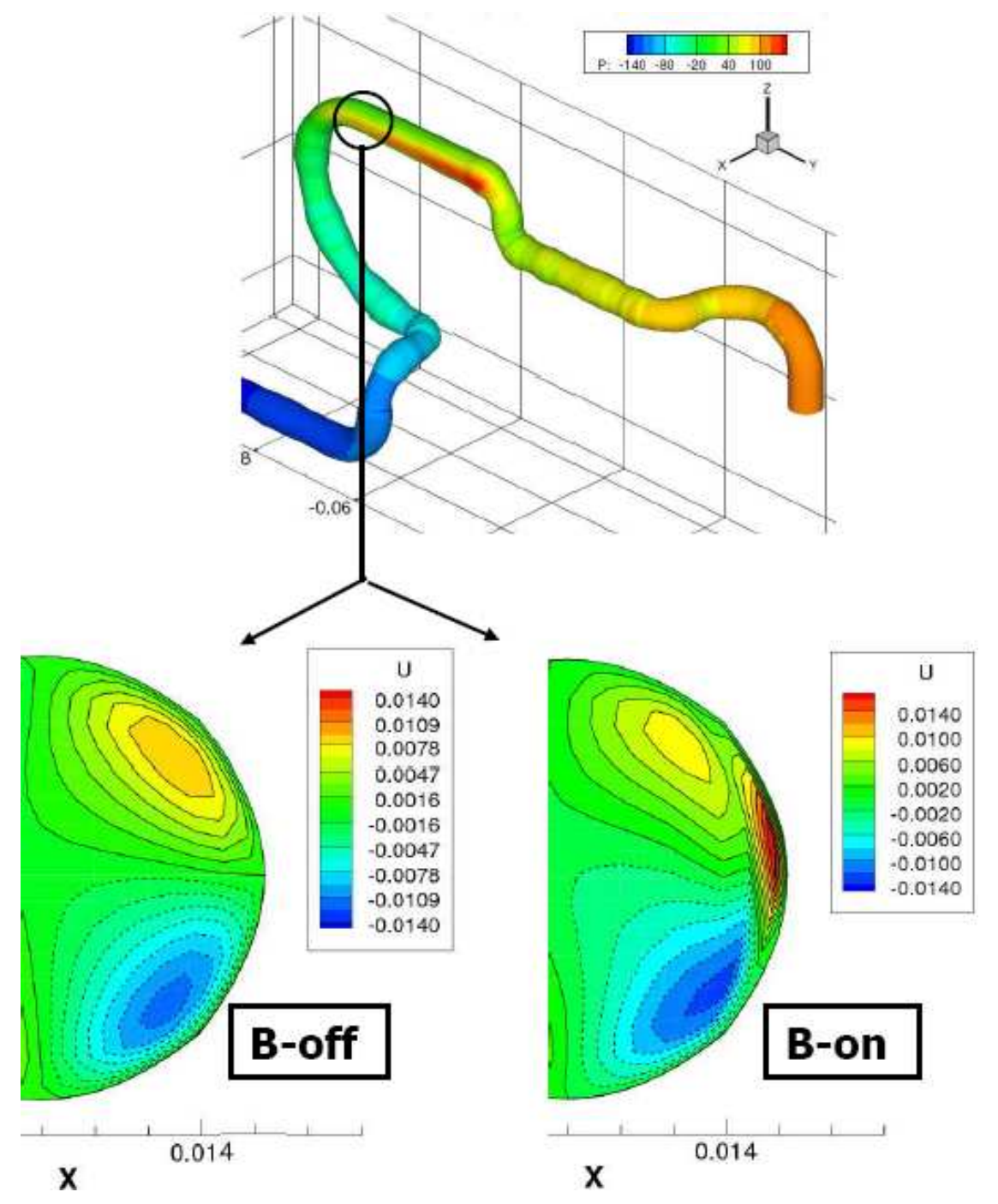

Figure 12: 